\title{
Fenómeno miotónico orofacial en pacientes con distrofia miotónica de Steiner
}

\section{Orofacial myotonic phenomenon in Steiner's myotonic dystrophy patients}

Guimarães AS*, Suazo GI**, Nagahashi Marie SK***

\section{RESUMEN}

La distrofia miotónica de Steinert (DM1) es una enfermedad hereditaria, caracterizada por desórdenes multisistémicos asociados a disfunción muscular. Una de las manifestaciones clínicas más frecuentes es el fenómeno miotónico, en el cual se produce un enlentecimiento de la relajación muscular postisométrica. En este estudio analizamos la presencia del fenómeno miotónico (FM) en movimientos de apertura cierre oral, movimientos de la lengua y de otros músculos periorales, en 50 pacientes adultos con diagnóstico molecular de DM1. Nosotros encontramos FM en los movimientos analizados en 32 de los 50 pacientes con DM1 analizados (64\%), dentro de los cuales, la mayor prevalencia afectó a los movimientos de la lengua, 21 casos $(65,6 \%)$, seguidos de los movimientos de apertura oral que afectó a 14 pacientes y de los movimientos de cierre oral que presentó fenómeno miotónico en 7 pacientes. Sólo 2 pacientes expresaron el signo en apertura y cierre oral y en 1 caso se encontró afectada la actividad del músculo buccinador. Este estudio muestra que los músculos de la región orofacial son frecuentemente afectados en los pacientes con DM1, por lo que el clínico debe reconocer el FM en estos pacientes.

Palabras clave: Distrofia miotónica de Steinert, distrofia muscular, miotonía.

\section{SUMMARY}

Steinert's myotonic dystrophy (DM1) is a hereditary disease, characterized by multisystem disorders associated with muscle dysfunction. One of the most frequent clinical manifestations is the myotonic phenomenon (MP), which produces a slowdown of post-isometric muscle relaxation. In this study, the presence of MP in the opening and closing oral movements, movements of the tongue, and other perioral muscles in 50 adult patients with DM1 molecular diagnostics was analyzed. We found MP in 32 of the 50 patients analyzed with DM1 (64\%), among which 21 cases (65.6\%) were affected with impaired movements of the tongue (highest prevalence), followed by the oral opening movements, which affected 14 patients, and oral closing movements presented in 7 patients. Only 2 patients expressed the symptom of oral opening and closing movements, and in 1 patient, buccinator muscle activity was observed. This study demonstrates that the muscles in the orofacial region are frequently affected in patients with DM1, and hence, the clinician should recognize the MP in these patients.

Key words: Steinert's myotonic dystrophy, muscular dystrophy, myotonia.

Fecha de recepción: 2 de marzo de 2010.

Aceptado para publicación: 6 de marzo de 2010.

* Instituto da Cabeça. Universidad Federal de São Paulo. Escola Paulista de Medicina. São Paulo. Brazil.

** Unidad de Anatomía Normal. Facultad de Ciencias de la Salud. Universidad de Talca. Chile.

*** Departamento de Neurología. Universidad de São Paulo. Brazil.

Guimarães Antonio S, Suazo Ivan G, Suely K.Marie. Fenómeno miotónico orofacial en pacientes con distrofia miotónica de Steiner. Av. Odontoestomatol 2010; 26 (3): 139-142. 


\section{INTRODUCCIÓN}

La distrofia miotónica de Steinert (DM1) es una enfermedad multisistémica que se transmite de manera autosómica dominante (1), de baja prevalencia en la población $(2,3)$ y que puede desarrollarse en cualquier período de la vida, con una mayor incidencia entre los 20 y 49 años (4). Los pacientes suelen ser diagnosticados de manera tardía debido al deterioro progresivo de la función muscular

En el sistema estomatognático la DM1 se acompaña de alteraciones en el crecimiento facial, relacionadas con un menor modelamiento muscular del desarrollo (5), dificultades de habla (6) y un aumento del riesgo de caries, gingivitis y desordenes de la articulación temporomandibular (7), por lo cual el odontólogo debe ser capaz de reconocer esta enfermedad.

Una de los signos característicos, de utilidad para el diagnóstico, es el denominado fenómeno miotónico, que se manifiesta como dificultad en la relajación muscular, consecuentemente, con una disminución en la velocidad de ejecución de los movimientos voluntarios. Existen escasos reportes de fenómenos miotónicos en los músculos masticadores (5). Para la práctica odontológica resulta relevante conocer el fenómeno miotónico en las estructuras musculares del sistema estomatognático, debido a que la aparición del enlentecimiento de la actividad muscular, puede estar enmascarada con posiciones antiálgicas o con dificultades mecánicas en los movimientos de apertura y cierre oral, como ocurre en desplazamiento anterior del disco de la ATM, siendo necesario su diagnóstico diferencial (8).

El objetivo de este estudio es analizar la prevalencia del fenómeno miotónico en el sistema estomatognático, en 50 pacientes con DM1.

\section{MATERIAL Y MÉTODOS}

El estudio fue aprobado por la comisión de ética e investigación del Hospital das Clínicas de la Facultad de Medicina de la Universidad de São Paulo (HCFMUSP). Los pacientes fueron voluntarios, informados de la naturaleza del estudio y dejaron constancia escrita de su consentimiento.
Participaron en este estudio 50 pacientes, 24 mujeres (media 38,63 años; SD 14,425) y 26 hombres (media 36,35; SD 12,093), con diagnóstico molecular de DM1 realizado con el método de Southern blot (9), atendidos en el HC-FMUSP. Los pacientes no presentaban otras enfermedades neurológicas asociadas.

Se analizó la presencia de fenómeno miotónico en los músculos de la masticación y en otros músculos de la cavidad oral. Se consideró que el paciente presentaba fenómeno miotónico si tardaba más de 0,5 segundos en iniciar el cese de la actividad de los músculos evaluados.

\section{Fenómeno miotónico en apertura}

Los pacientes realizaron contracción isométrica en máxima intercuspidación de los músculos elevadores mandibulares, durante 10 segundos. Después de ese período se pidió a los pacientes que realizaran apertura oral.

\section{Fenómeno miotónico en cierre}

Los pacientes realizaron apertura oral máxima, que luego mantuvieron por 10 segundos, por contracción isométrica de los músculos depresores mandibulares. Posteriormente período se pidió a los pacientes que realizaran cierre oral.

\section{Fenómeno miotónico en otros músculos de la cavidad oral}

Mediante evaluación funcional, se analizó la presencia de retardo en la relajación de los músculos faciales y de la lengua. Los músculo faciales se evaluaron mediante la expresión facial y los músculos de la lengua se analizaron como grupo con movimientos de protrusión y retracción lingual.

\section{RESULTADOS}

La presencia de fenómeno miotónico en alguno de las funciones del sistema estomatognático analizadas se encontró en 32 de los 50 pacientes con DM1 
analizados (64\%), dentro de los cuales, la mayor prevalencia afectó a los movimientos de la lengua, 21 casos (65.6\%), seguidos de los movimientos de apertura oral que afectó a 14 pacientes y de los movimientos de cierre oral que presentó fenómeno miotónico en 7 pacientes. Sólo 2 pacientes expresaron el signo en apertura y cierre oral y en 1 caso se encontró afectada la actividad del músculo buccinador.

En la mayoría de los pacientes que expresaron fenómeno miotónico en apertura y cierre también se encontraba alterado el movimiento de la lengua.

El detalle de la distribución del fenómeno miotónico en los 32 pacientes que expresaron el signo en los movimientos del sistema estomatognático analizados se encuentran en la Tabla 1.

\section{DISCUSIÓN}

En este estudio encontramos una alta incidencia de fenómeno miotónico orofacial en las funciones de apretura y cierre y movimientos linguales, lo que se manifiesta en un enlentecimiento del movimiento que dificulta la colaboración del paciente en la atención odontológica y en el cuidado de su salud oral, por lo que la DM1 actúa como factor de riesgo para el desarrollo de caries y gingivitis (10). La mayoría de los pacientes que presentaron el fenómeno miotónico tenían algún compromiso lingual, lo que implica alteraciones en la fonoarticulación que puede confun- dirse con desórdenes mentales y disminuyen la autoestima del paciente.

La presencia del fenómeno miotónico sugiere cambios morfológicos importantes en los músculos masticadores que implican disminución de la fuerza muscular, posiblemente asociada al reemplazo de fibras musculares y de los husos neuromusculares por tejido adiposo y el aumento del tejido conectivo entre las fibras musculares (10). La alteraciones originadas por la disminución de un importante input propioceptivo muscular pueden verse aumentadas en estos pacientes por la pérdida de piezas dentarias, generando inestabilidad mandibular y dislocación de la ATM $(8,12,13)$.

En conclusión, este estudio muestra que los músculos de la región orofacial están frecuentemente afectados en los pacientes con DM1, por lo que el clínico debe reconocer el fenómeno miotónico, especialmente en casos en los cuales la expresión de la enfermedad ocurre en la vida adulta, como signo precoz del desarrollo de la DM1.

\section{BIBLIOGRAFÍA}

1. Junien C. Myotonic dystrophy of Steinert. J Genet Hum 1989;37:51-4.

2. Darin N, Tulinius M. Neuromuscular disorders in childhood:a descriptive epidemiological study from western Sweden. Neuromuscul Disord 2000;10:1-9.

\begin{tabular}{|lccccc|}
\hline \multicolumn{2}{|c|}{$\begin{array}{c}\text { TABLA 1.- FENÓMENO MIOTÓNICO (FM) EN LOS 32 PACIENTES CON DMS QUE } \\
\text { EXPRESARON EL SIGNO EN LOS MOVIMIENTOS DEL SISTEMA ESTOMATOGNÁTICO } \\
\text { ANALIZADOS }\end{array}$} \\
\hline & $\begin{array}{c}\text { FM en } \\
\text { apertura }\end{array}$ & $\begin{array}{c}\text { FM en } \\
\text { cierre }\end{array}$ & $\begin{array}{c}\text { FM en } \\
\text { otros músculos } \\
\text { de la cavidad oral }\end{array}$ & $\begin{array}{c}\text { FM en } \\
\text { la lengua }\end{array}$ & $\begin{array}{c}\text { FM en } \\
\text { apertura } \\
\text { y cierre }\end{array}$ \\
\hline Manifestación única & 6 & 4 & - & 8 & 1 \\
\hline $\begin{array}{l}\text { Acompañado de FM } \\
\text { en la lengua }\end{array}$ & 8 & 3 & 1 & - & 1 \\
\hline Total & 14 & 7 & 1 & 8 & 2 \\
\hline
\end{tabular}


3. Harper PS. Myotonic dystrophy. 3rd ed. Saunders: Philadelphia; 2001.

4. Mladenovic J, Pekmezovic T, Todorovic S, Rakocevic-Stojanovic V, Savic D, Romac S, Apostolski S. Epidemiology of myotonic dystrophy type 1 (Steinert disease) in Belgrade (Serbia). Clin Neurol Neurosurg 2006;108: 757-60.

5. Zanoteli E, Yamashita HK, Suzuki H, Oliveira AS, Gabbai AA. Temporomandibular joint and masticatory muscle involvement in myotonic dystrophy: a study by magnetic resonance imaging. Oral Surg Oral Med Oral Pathol Oral Radiol Endod 2002;94:262-71.

6. Penarrocha M, Bagan JV, Vilchez J, Millian MA, Fernandez $S$. Oral alterations in Steinert's myotonic dystrophy: a presentation of two cases. Oral Surg Oral Med Oral Pathol 1990;69:698700.

7. Sjogreen L, Engvall M, Ekstrom AB, Lohmander A, Kiliaridis S , Tulinius M. Orofacial dysfunction in children and adolescents with myotonic dystrophy. Dev Med Child Neurol 2007;49:18-22.

8. Friedman RD, Joe J, Bodak LZ. Myotonic dystrophy: report of a case. Oral Surg Oral Med Oral Pathol 1980;50:229-32.
9. Southern EM. Detection of specific sequences among DNA fragments separated by gel electrophoresis. J Mol Biol 1975;98:503-17.

10. Engvall M, Sjogreen L, Kjellberg H, Robertson A, Sundell S, Kiliaridis S. Oral health in children and adolescents with myotonic dystrophy. Eur J Oral Sci 2007;115:192-7.

11. Bakke M, Kirkeby S, Jensen BL, Hansen HJ, Kreiborg S, Hjorting-Hansen E, Michler L, Moller E. Structure and function of masticatory muscles in a case of muscular dystrophy. J Oral Pathol Med 1990;19:335-40.

12. Gold GN. Temporomandibular joint dysfunction in myotonic dystrophy. Neurology 1966;16:212-6.

13. Wilson A, Mackay L, Ord RA. Recurrent dislocation of the mandible in a patient with myotonic dystrophy. J Oral Maxillofac Surg 1989; 47:132932.

\section{CORRESPONDENCIA}

Antonio S. Guimarães

Rua Visconde da Luz, 60/31

04537070 São Paulo SP. Brazil

e-mail: asgatm@uol.com.br 